\title{
Effect of exercise on the caloric intake of breast cancer patients undergoing treatment
}

\author{
C.L. Battaglini ${ }^{1}$, J.P. Mihalik¹, M. Bottaro², C. Dennehy ${ }^{3}$, M.A. Petschauer ${ }^{1}$, \\ L.S. Hairston ${ }^{1}$ and E.W. Shields ${ }^{1}$ \\ 1Department of Exercise and Sport Science, University of North Carolina at Chapel Hill, Chapel Hill, \\ NC, USA \\ ${ }^{2}$ Faculdade de Educação Física, Universidade de Brasília, Brasília, DF, Brasil \\ ${ }^{3}$ Navitas Cancer Rehabilitation Centers of America, Inc., Westminster, CO, USA
}

Correspondence to: C. Battaglini, Department of Exercise and Sport Science, University of North Carolina at Chapel Hill, 313 Woollen Gym, Campus Box 8605, Chapel Hill, NC 27599-8605, USA

E-mail: claudio@email.unc.edu

\begin{abstract}
The purpose of this study was to examine the effects of an exercise intervention on the total caloric intake (TCl) of breast cancer patients undergoing treatment. A secondary purpose was to determine whether or not a relationship existed between changes in $\mathrm{TCl}$, body fat composition (\%BF), and fatigue during the study, which lasted 6 months. Twenty females recently diagnosed with breast cancer, scheduled to undergo chemotherapy or radiation, were assigned randomly to an experimental $(N=10)$ or control group $(\mathrm{N}=10)$. Outcome measures included TCl (3-day food diary), \%BF (skinfolds), and fatigue (revised Piper Fatigue Scale). Each exercise session was conducted as follows: initial cardiovascular activity (6-12 min), followed by stretching (5-10 min), resistance training (15-30 $\mathrm{min}$ ), and a cool-down (approximately $8 \mathrm{~min}$ ). Significant changes in TCl were observed among groups $\left(F_{1,18}=8.582 ; \mathrm{P}=0.009\right)$, at treatments 2 and 3 , and at the end of the study [experimental $(1973 \pm 419)$, control (1488 \pm 418$)$; experimental (1946 \pm 437$)$, control (1436 \pm 429$)$; experimental $(2315 \pm 455)$, control (1474 \pm 294$)$, respectively]. A significant negative correlation was found (Spearman rho (18) $=-0.759 ; \mathrm{P}<0.001$ ) between TCl and \%BF and between TCl and fatigue levels (Spearman rho $(18)=-0.541 ; P=0.014$ ) at the end of the study. In conclusion, the results of this study suggest that an exercise intervention administered to breast cancer patients undergoing medical treatment may assist in the mitigation of some treatment side effects, including decreased $\mathrm{TCl}$, increased fatigue, and negative changes in body composition.
\end{abstract}

Key words: Exercise; Breast cancer; Caloric intake; Fatigue; Body composition

M. Bottaro was the recipient of a productivity grant from CNPq (\#476206/2007-3).

Received December 13, 2007. Accepted August 15, 2008

\section{Introduction}

Breast cancer is the second leading cause of cancerrelated death among women. While breast cancer is ranked third among the most common cancers present in society, it is ranked fifth among overall cancer-related deaths (1). At present, there are approximately 10.5 million Americans with a cancer history and 2.4 million of those are breast cancer survivors (2). An estimated 211,240 new cases of invasive breast cancer were diagnosed among women in the USA in 2005 (3). Modern technology advancements, greater education, significant improvements in adjuvant chemotherapies, and self-examinations have contributed to the increased survival rate among breast cancer patients.

Reduced capacity for work and being less able to efficiently complete tasks, both of which are often accompanied by weariness and tiredness, are conditions characteristic of cancer-related fatigue (4). While the exact etiol- 
ogy of cancer-related fatigue is not fully understood, it is thought to be based on the physiological and psychological effects of cancer treatments. Regardless of treatment type, the consistent and often persistent manifestation of cancer treatment-related fatigue is pervasive.

There have been many theories concerning the factors that contribute to cancer-related fatigue. One, for example, suggests that a reduced blood count caused by chemotherapy could lead to anemia that results in fatigue $(5,6)$. Other factors include medications, psychological distress, changes in immune function, excessive inactivity, neuromuscular dysfunction, and cognitive factors. The present study, however, has focused attention on data that suggest that the body's inability to efficiently process nutrients, notable changes in the body's nutritional requirements during and following treatment, and a decrease in overall energy intake could be significant contributing factors to the specific fatigue experienced by cancer patients undergoing treatment (7). Progressive weight loss and the rate at which weight is lost during treatment have been associated with survivorship rates (8). In addition, negative changes in body composition have been related to poor responses to chemotherapy and an overall decrease in quality of life (8).

Exercise has been shown to have a positive physiological and psychological impact on mitigating some of the side effects associated with treatment modalities, including positive changes in body composition and fatigue levels $(1,7)$. Energy loss usually observed during treatment has been shown to improve with an aerobic training program (9). Results from studies that explored aerobic and resistance training as the exercise modes showed positive effects on decreasing fatigue levels, improvement in quality of life, and muscle strength $(7,8,10,11)$.

Even though the majority of studies involving aerobic and resistance exercise have reported improvements in aerobic capacity, muscle strength, body composition, and fatigue $(12,13)$, the impact of exercise on caloric intake, usually known to be altered in patients undergoing treatment, has not been fully explored. Furthermore, the relationship between changes in caloric intake, body composition, and fatigue levels has not been explored in previous studies that involved exercise as a complementary therapy aimed to assist in the mitigation of side effects of cancer treatment. Therefore, the purpose of this study was to examine the effects of an individualized prescriptive exercise intervention on total caloric intake (TCl) in breast cancer patients undergoing chemotherapy or radiation treatment. A secondary purpose was to determine whether or not a relationship existed between changes in $\mathrm{TCl}$, body composition, and fatigue during treatment.

\section{Material and Methods}

\section{Subjects and experimental design}

This is a randomized controlled trial study [experimental (exercise) and control (no exercise)] developed and conducted in northern Colorado. TCl was assessed at six intervals during the course of the study. Pre- (post-surgery) and post-intervention (final assessment performed at the end of the study) measures of cardiovascular endurance, dynamic muscular endurance, body composition (percent body fat, \%BF), and fatigue levels were recorded and included in the design.

Twenty female subjects with ages ranging from 35-70 years who had been recently diagnosed with having breast cancer volunteered for this study. The following criteria were used for determining whether or not an individual was included in the study: a) recent breast cancer diagnosis; b) designated to undergo any type of surgery; c) required to receive either chemotherapy or radiation, or a combination of the two after surgery; d) age between $35-70$ years during the course of the study. If cardiovascular disease, acute or chronic respiratory disease, acute or chronic bone, joint, or muscular abnormalities were observed during the medical history review and physical examination by an oncologist, and if it was determined that the volunteer would not be able to engage in regular exercise, the patient was informed and excluded from the study. Patient's characteristics are presented in Table 1.

After the screening process for participation and explanations regarding the study, the study protocol was reviewed and participants decided if they wished to participate in the study. The protocol and an informed consent form approved by the University of Northern Colorado's Internal Review Board (UNCO IRB) and the North Colorado Medical Center's Internal Review Board (NCMC IRB) were signed by each participant. According the Health Insurance Portability and Accountability Act of 1996 (HIPAA), all subjects recruited after April 13, 2003 were asked to sign the HIPAA authorization to use or disclosure protected health information for research prior to enrollment in the study.

Table 1. Characteristics of breast cancer patients who participated in the study.

\begin{tabular}{lcc}
\hline & Control group & Exercise group \\
\hline Age (years) & $56.6 \pm 16$ & $57.5 \pm 23$ \\
Body weight $(\mathrm{kg})$ & $81.7 \pm 25.0$ & $77.3 \pm 27.3$ \\
Height $(\mathrm{cm})$ & $169.2 \pm 10.2$ & $168.9 \pm 10.2$ \\
Body fat $(\%)$ & $29.84 \pm 14.9$ & $29.13 \pm 10.1$ \\
\hline
\end{tabular}

Data are reported as means \pm SD for 10 subjects in each group. 


\section{Assessment protocols}

Patients were assigned randomly to two different groups the week after diagnosis and before surgery. Before surgery, the patients were assessed for $\mathrm{TCl}$, fatigue levels, body composition (skinfolds) and fitness, which was comprised of cardiovascular endurance and dynamic muscular endurance tests. During the study, which lasted 6 months, $\mathrm{TCl}$, body composition, and fatigue levels were assessed 6 times throughout the investigation (pre-surgery, post-surgery, at treatments $1,2,3$, and at the end of the study) and 2 fitness assessments were administered (post-surgery, and at the end of the study). During treatment, the assessments were performed at regular intervals at approximately 4 weeks apart according to each patient's treatment schedule. Because some patients were still receiving treatment during week 21 , fitness assessments were administered a day other than that of the subject's scheduled treatment. If a patient was to receive only radiation, their treatment began at week 5 , with their first assessment being administered at week 6 , followed by weeks 10 and 14, and at the end of the study on the 21st week. All patients were assessed at 4-week intervals whether or not they began their treatment at week 5 as mentioned previously.

\section{Total caloric intake analysis}

A 3-day food diary was used to record the amount of caloric intake as determined by the recommendations of the nutritional assessment guideline by Lee and Nieman (14). The data were analyzed to determine whether TCI had changed enough throughout the experiment to have an effect on body composition. Patients were asked to record both food and beverage consumption (including snacks) for 2 days during the week and 1 day during the weekend.

\section{Body composition analysis}

The body composition of each patient was assessed using skinfold measurement for the determination of \%BF. Large skinfold calipers (Cambridge Scientific Industries, Inc., USA) were used for the determination of body composition. Skinfold measurements were performed using the 3-site skinfold measurement formula for women (15). For patients who had undergone transverse rectus abdominis muscle flap reconstructive surgery, triceps, thigh and suprailiac sites were used. The body composition assessment was performed approximately 30 min prior to administration of cancer treatment by the oncologist.

Self-reported fatigue analysis

Fatigue levels were assessed using the revised Piper et al. (16) Fatigue Scale (PFS). This 22-item self-reported scale measures fatigue on a scale of 0 to 10 , as well as 4 subjective fatigue domains: affective, sensory, cognitive, and behavioral, yielding a total fatigue score. The majority of cancer patients experience the highest levels of fatigue approximately 3 days following treatment. Patients were, therefore, asked to answer questions on the PFS during the last day of the week before they reported to the next scheduled assessment/treatment.

\section{Fitness assessment}

The assessment of cardiovascular endurance and dynamic muscular endurance were included in the fitness assessments. Heart rate responses were determined by each patient wearing a heart rate monitor throughout the cardiovascular assessments and the control of intensity during exercise sessions. Fitness assessments were performed at the Rocky Mountain Cancer Rehabilitation Institute at the University of Northern Colorado (Greeley, CO, USA). Cardiovascular endurance assessments were performed on a Quinton model 65 treadmill (USA). Cardiovascular endurance $\left(\mathrm{VO}_{2 \max }\right)$ was assessed using the modified Bruce treadmill protocol. The test, which is a submaximal exercise test, is often used for high-risk populations because of the low level of stress it imposes on patients (17).

The following exercises were utilized to assess the dynamic muscular endurance of each subject: leg extension, seated leg curl, lateral pull down, and seated chest press. The assessments were performed on LifeFitness (USA) and Quantum (USA) weight machines. A submaximal endurance protocol that required the use of free weights helped predict one maximal repetition for each subject. This submaximal endurance protocol was developed for middle-aged and older women (18).

\section{Exercise intervention}

The exercise intervention assigned to the experimental group began in week 6 of the study and lasted until the conclusion of the study following week 21 . The exercise group began their routine approximately 3 weeks prior to receiving their first cancer treatment due to the post-cancer surgery treatment schedule. Exercise sessions were held at the Rocky Mountain Cancer Rehabilitation Institute and/or at the University of Northern Colorado Campus Recreation Center at no cost to the patient. Patients assigned to the experimental group exercised 2 days a week for no longer than a 60 -min period. The rest time allowed between each session was no less than $48 \mathrm{~h}$ and no more than $84 \mathrm{~h}$. Each patient was assigned an individualized exercise intervention program designed in accordance 
with the recommendations of the American College of Sports Medicine exercise guidelines for elderly populations (19) and guidelines published in Exercise and Cancer Recovery (20). Intensities varying from $40-60 \%$ of the patient's predicted maximum exercise capacity were assigned to each activity and were based on the results of the first fitness assessment administered prior to surgery.

Each exercise session was supervised by an undergraduate or graduate cancer exercise specialist from the Department of Sport and Exercise Science, University of Northern Colorado. The exercise training included cardiovascular, resistance, and flexibility training. Each exercise session was conducted as follows: initial cardiovascular activity (6-12 $\mathrm{min})$, followed by a stretching session (5-10 $\mathrm{min})$, resistance training (15-30 $\mathrm{min}$ ), and a cool-down that included stretching activities (approximately $8 \mathrm{~min}$ ).

The intensities used for the resistance training program were determined by the first fitness assessment (18). The protocol was used to predict the highest level of intensity for one maximum repetition during a sub-maximal endurance test for middle- to older-aged women. All major muscle groups were utilized during the resistance training sessions. The exercises were performed using the LifeFitness and Quantum weight machines, free weights, elastic bands, or therapeutic balls. The resistance exercises were as follows: lateral and front raises, triceps extension, leg press, leg extension, leg curl, standing calf raises, horizontal chest press, lateral pull down, alternating bicep curls with dumbbells, regular crunches, oblique crunches, and lower abdominal crunches.

Patients assigned to the exercise group increased the number of sets performed each week, which never exceeded three sets of each exercise. During the first week, subjects assigned to the experimental group had to endure one set for each exercise performed during the sessions. Subjects assigned to the exercise group progressively increased to two then three sets per exercise by the end of the study. Movements during each exercise were performed at moderate speed (3s of concentric phase and 3 $s$ of the eccentric phase of the movement during each repetition for each exercise). Rest time between each set and each exercise varied according to the subject's desires, but was approximately $1 \mathrm{~min}$.

Cardiovascular activities included the following: walking on a treadmill [Precor C966 (USA), Quinton 65, or LifeFitness TR $9500 \mathrm{HR}$, the use of the cycle ergometer (LifeFitness $8500 \mathrm{HR}$, or elliptical equipment LifeFitness CT $9500 \mathrm{HR}$ or Precor EFX 546). Exercise training programs have been shown to improve the overall quality of life, level of fitness, improved levels of self-esteem, and confidence of cancer patients (9). It has also been sug- gested that while controlling energy intake, it may be possible to increase energy expenditure through exercise (21). Each exercise session was modified according to the patient's physical state prior to the exercise session. On the days in which patients were experiencing extreme fatigue the exercise session intensity and duration were modified.

\section{Statistical analysis}

All data were analyzed using the statistical software program SPSS, version 14.0, for Windows. Statistical significance was determined with a probability value less than or equal to 0.05 . For the assessment of changes and differences in $\mathrm{TCl}$ between the exercise and control groups, a $2 \times 6$ mixed model repeated measures ANOVA was used. $\mathrm{TCl}$ measurements used for the analyses included measurements obtained prior to surgery, post-surgery, during treatments $1,2,3$, and at the end of the study (final assessment). Tukey HSD post hoc analyses were performed to determine at which time during treatment significant differences between groups were observed. For the correlation analyses relating $\mathrm{TCl}, \% \mathrm{BF}$, and fatigue levels in both the exercise and control groups during the study, a Spearman rho correlation was employed. The variables included in the model were the change score for $\mathrm{TCl}$ and for \%BF, and fatigue levels. The change in $\mathrm{TCI}, \% \mathrm{BF}$, and fatigue were determined as the difference between the results of the final assessment compared to the initial assessment.

\section{Results}

A significant time versus group interaction was present $\left(F_{4.346,78.221}=7.895 ; \mathrm{P}<0.001\right)$. In addition, the main effect for group was significant $\left(F_{1,18}=8.582 ; \mathrm{P}=0.009\right)$ indicating that the exercise group $(\mathrm{TCl}=1998.531 \pm 451.65 \mathrm{kcal})$ had a significantly higher $\mathrm{TCl}$ than our patients in the control group $(\mathrm{TCl}=1597.782 \pm 359.08 \mathrm{kcal})$ (Figure 1). The effect for time was marginally significant $\left(F_{4.346,78.221}=\right.$ 2.084; $P=0.085$ ).

After combining the exercise and control groups into one, a relationship between $\mathrm{TCl}$ and \%BF was explored. A negative correlation was found $(r h o(18)=-0.759 ; \mathrm{P}<$ 0.001 ), indicating a significant linear relationship between the two variables. Patients with a greater change in $\mathrm{TCl}$ (higher during final assessment compared to pre-surgery) tended to have lower changes in \%BF.

Using the same procedure, the relationship between the change in $\mathrm{TCl}$ and fatigue level (PFS) was also analyzed using a Spearman rho correlation. A negative correlation was found $(r h o(18)=-0.541 ; P=0.014)$, indicating a 
significant relationship between $\mathrm{TCl}$ and fatigue. Patients with a greater change in $\mathrm{TCl}$ tended to have greater changes in fatigue level (fatigue level was lower during final assessment than at pre-surgery).

\section{Discussion}

This study was designed to examine the impact of exercise on $\mathrm{TCl}$ in breast cancer patients undergoing chemotherapy or radiation treatment. The results of the current study suggest that an individualized prescriptive exercise program including aerobic, flexibility, and resistance training helps to increase breast cancer patient's overall $\mathrm{TCl}$ while receiving treatment. Patients placed in the exercise group experienced an increase in $\mathrm{TCl}$ of $20 \%$ while the control group experienced a decrease of approximately $7 \%$. The significant increase of $\mathrm{TCl}$ demonstrated by the exercise group could be due to the impact of exercise on fatigue, nausea, and the body's physiological need for nourishment following participation in physical activity.

The increased caloric intake experienced by the exercise group may have contributed to the elevated energy levels reported by patients in this group and thus reducing the feeling of fatigue experienced prior to engaging in the exercise regimen. Another finding of great importance was the relationships observed between $\mathrm{TCl}$ and \%BF. The results of the current study are somewhat in agreement with a study conducted by Winningham and MacVicar (22). They examined the impact on nausea of a 10-week aerobic exercise program administered three times per week. The population included in the study was breast cancer patients undergoing chemotherapy. A group of patients were randomized to either an exercise (aerobic training), a placebo (light flexibility workouts) or to a control non-exercise group. Significant improvements were observed in the exercise group but not in the placebo or control groups. These investigators concluded that the impact of aerobic exercise did, in fact, reduce nausea in the aerobic exercise group and that aerobic training could be safely implemented during treatment. Since nausea is a common symptom experienced by cancer patients during treatment, Winningham and MacVicar's study showed that this type of exercise can reduce symptoms of nausea during treatment. Even though the current study did not assess nausea during treatment, it is believed that the impact of the exercise intervention used in the current study may have impacted nausea and consequently allowed patients in the exercise group to experience a higher caloric intake compared to the control group.

One of the major issues in the breast cancer population is the increase in body weight during treatment. This increase in body weight is usually followed by increased \%BF and reduced lean body mass. The increase in adiposity is significantly associated with decrease survival and re-occurrence rates (23). Furthermore, in a large cohort study, Tretli et al. (24) found that the association between obesity and poor breast cancer outcome was of extreme importance. In that study, women diagnosed with stage I breast cancer who were in the upper quintile of body mass index were $70 \%$ more likely to dye from breast cancer than those below this level. Even though an increase in body weight usually occurs during palliative care, during treatment, decreased TCI can induce loss of lean tissue with increases in \%BF that can decrease overall functionality, tremendously affecting the ability of patients to perform even the simple daily tasks. The present study is the first that has explored the impact of exercise on the relationship between $\mathrm{TCl}$ and body composition in breast cancer patients undergoing treatment.

The second purpose of this study was to determine the extent of the relationship between $\mathrm{TCl}$, body composition, and fatigue throughout treatment. Our findings indicated that breast cancer patients undergoing treatment who engage in physical activity experience a higher caloric intake throughout the duration of treatment than those who engage in little to no physical activity while decreasing \%BF and fatigue levels compared to patients who do not engage in regular physical activity. Galvao and Newton (1) reviewed many studies that used exercise as an intervention to mitigate side effects of cancer treatment and they found quite a few studies in which body composition of patients in an exercise group either maintained body weight and body composition or improved body composition significantly.

Winningham et al. (25) conducted a study to determine the effects of aerobic training on body composition in breast

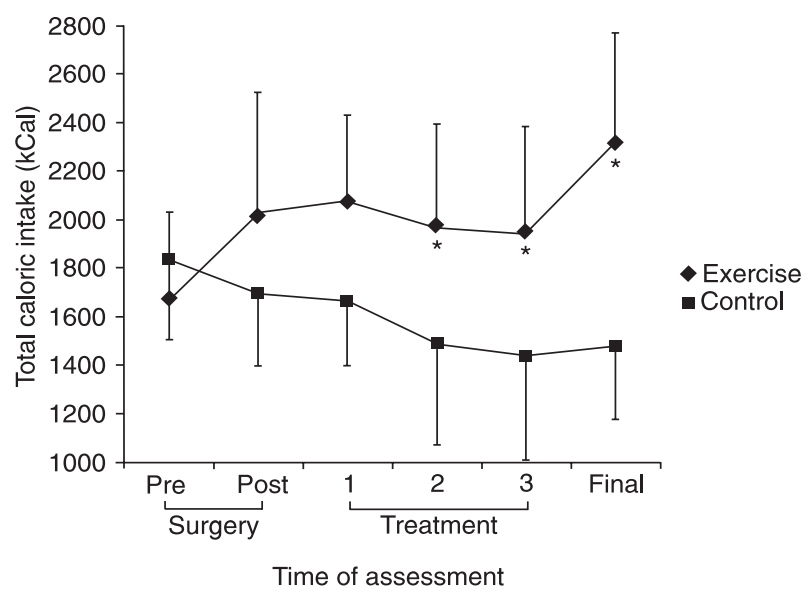

Figure 1. Effect of exercise on total caloric intake of breast cancer patients under treatment. The figure describes a period of 24 weeks. ${ }^{*} \mathrm{P} \leq 0.05$ compared to control group $(2 \times 6$ mixed model repeated measures ANOVA). 
cancer patients undergoing chemotherapy. Patients were assigned to an exercise group that performed aerobic training for 10-12 weeks. The subjects exercised at a $60-80 \%$ intensity of maximal heart rate. Using the skinfold measurement, they reported increased lean body mass and decreased fat tissue for the subjects who exercised. Similarly, the present study found improvement in body composition. The major difference with the Winningham study was that an exercise intervention composed of aerobic, resistance training, and flexibility was used in the current study.

Studies examining the impact of exercise on fatigue levels of breast cancer patients have also shown great improvement compared to baseline results or control groups. In three studies conducted by Schwartz and colleagues (26-28), breast cancer patients engaged in 4 weeks of aerobic training consisting of walking showed a significant decrease in overall fatigue levels. Mock et al. (29) using a similar but longer duration protocol (5-6 weeks) also found significant reduction of fatigue levels. A study conducted by Segal et al. (30) using a protocol consisting of resistance training as the primary form of exercise and a prostate cancer population also found a significant decrease in overall fatigue levels. The current study used a combination of aerobic, resistance, and flexibility workouts as the intervention. In agreement with the studies cited above, a significant reduction in fatigue levels was also observed in the exercise group but not in the control group.

Besides confirming similar results described in the literature, the present study also detected a significant relationship between both \%BF and fatigue levels and TCl. The improvement in body composition and the relationship between \%BF and $\mathrm{TCl}$ was a great surprise. Although overall body weight was maintained in the exercise group throughout the experiment, the resulting decrease in \%BF indicated the significance of exercise intervention in promoting lean body mass. This correlation suggests that exercise programs performed in conjunction with cancer patients undergoing treatment in this study were sufficient to cause a positive change in body composition regardless of the increase in TCl. Breast cancer patients undergoing treatment are known to either gain or lose weight, sometimes resulting in obesity or cancer-mediated cachexia. The reduction in \%BF while maintaining body weight observed as a result of the exercise program administered throughout the current study are positive indicators of the impact of exercise on cancer patients while receiving treatment and should be further explored. If the results of the current study can be reproduced in a larger trial, exercise may turn into a powerful intervention to assist patients to maintain or improve their body weight/body composition, creating opportunities for better treatment outcomes and improvement in survival.
Fatigue experienced by cancer patients has been described as a process in which both psychological and physiological changes during and after the administration of cancer treatments lead patients to experience a decrease in their overall functionality (9). There was a significant inverse relationship observed in the current study demonstrating an increase in $\mathrm{TCl}$ paired with a reduction in fatigue levels in patients assigned to the exercise group. The development of fatigue in breast cancer patients is believed to be caused by numerous factors. The results of the current study, however, suggest that $\mathrm{TCl}$ becomes an important factor in managing fatigue levels throughout treatment and therefore further investigations on caloric intake during treatment for breast cancer patients should be explored.

Since weight changes are usually associated with poor diagnosis and treatment outcomes with adverse effects being associated with the risk for the development of cardiovascular disease and diabetes, and for breast cancer recurrence, and to increase chances for long-term survival, exercise seems to be a powerful and safe intervention for breast cancer patients. Breast cancer survivors should be advised to engage in healthy lifestyles to maintain healthy weight. Even if body weight cannot be maintained to a recommended level, research has shown that as little as $5-10 \%$ reduction in a period of 6-12 months is sufficient to elicit positive physiological changes, i.e., changes in elevated plasma lipids and high fasting levels of insulin (31). Breast cancer patients should be encouraged to maintain or even increase their body weight during treatment. After treatment, the maintenance of weight or safe weight reduction through exercise and healthy diet should be encouraged for those breast cancer survivors who gained more weight than expected. Moderate exercise, during and after treatment should be considered for the maintenance of lean muscle mass while avoiding excess body fat (32).

The results of this study suggest that the administration of exercise as treatment in breast cancer patients receiving chemotherapy may assist in the management of some side effects, including a decrease in $\mathrm{TCl}$, increased fatigue levels, and negative changes in body composition. Therefore, it is recommended that an individualized exercise prescription composed of aerobic, resistance, and flexibility training be suggested as a form of adjunct therapy to cancer treatment to improve $\mathrm{TCl}$, decrease fatigue levels, while also impacting positively changes in body composition and fatigue levels among breast cancer patients undergoing treatment.

\section{Acknowledgments}

We express our gratitude to the women who participated in the study. 


\section{References}

1. Galvao DA, Newton RU. Review of exercise intervention studies in cancer patients. J Clin Oncol 2005; 23: 899-909.

2. National Cancer Institute $(\mathrm{NCl})$. Dictionary of cancer terms. http://www.cancer.gov/templates/db_alpha.aspx? expand=B.

3. American Cancer Society (ACS). Breast cancer facts and figures 2005-2006. http:www.cancer.org.

4. Stasi R, Abriani L, Beccaglia P, Terzoli E, Amadori S. Cancer-related fatigue: evolving concepts in evaluation and treatment. Cancer 2003; 98: 1786-1801.

5. Andrews P, Morrow G, Hickok J, Roscoe J, Stone P. Mechanisms and models of fatigue associated with cancer and its treatment: evidence from preclinical and clinical studies. In: Armes J, Krishnasamy M, Higginson I (Editors), Fatigue in cancer. New York: Oxford Press; 2004. p 51-87.

6. Carteni G, Giannetta L, Ucci G, De Signoribus G, Vecchione A, Pinotti $G$, et al. Correlation between variation in quality of life and change in hemoglobin level after treatment with epoetin alfa 40,000 IU administered once-weekly. Support Care Cancer 2007; 15: 1057-1066.

7. Battaglini C, Bottaro M, Dennehy C, Rae L, Shields E, Kirk $D$, et al. The effects of an individualized exercise intervention on body composition in breast cancer patients undergoing treatment. São Paulo Med J 2007; 125: 22-28.

8. Tisdale MJ. Wasting in cancer. J Nutr 1999; 129: 243S$246 S$.

9. Dimeo F, Rumberger BG, Keul J. Aerobic exercise as therapy for cancer fatigue. Med Sci Sports Exerc 1998; 30: 475478.

10. Daley AJ, Crank H, Saxton JM, Mutrie N, Coleman R, Roalfe A. Randomized trial of exercise therapy in women treated for breast cancer. J Clin Oncol 2007; 25: 1713-1721.

11. Headley JA, Ownby KK, John LD. The effect of seated exercise on fatigue and quality of life in women with advanced breast cancer. Oncol Nurs Forum 2004; 31: 977983.

12. Courneya KS, Segal RJ, Mackey JR, Gelmon K, Reid RD, Friedenreich $\mathrm{CM}$, et al. Effects of aerobic and resistance exercise in breast cancer patients receiving adjuvant chemotherapy: a multicenter randomized controlled trial. J Clin Oncol 2007; 25: 4396-4404.

13. Segal R, Evans W, Johnson D, Smith J, Colletta S, Gayton $J$, et al. Structured exercise improves physical functioning in women with stages I and II breast cancer: results of a randomized controlled trial. J Clin Oncol 2001; 19: 657-665.

14. Lee R, Nieman D. Nutritional assessment. New York: McGraw Hill; 2003.

15. Jackson AS, Pollock ML, Ward A. Generalized equations for predicting body density of women. Med Sci Sports Exerc 1980; 12: 175-181.

16. Piper BF, Dibble SL, Dodd MJ, Weiss MC, Slaughter RE, Paul SM. The revised Piper Fatigue Scale: psychometric evaluation in women with breast cancer. Oncol Nurs Forum 1998; 25: 677-684.

17. Heyward V. Advanced fitness assessment and exercise prescription. Champaign: Human Kinetics; 1998.

18. Kuramoto K, Payne V. Predicting muscular strength in women: a preliminary study. Res Q Exerc Sport 1995; 66: 168-172.

19. American College of Sports Medicine. ACSM's guidelines for exercise testing and prescription. Baltimore: Williams \& Wilkins; 2000.

20. Schneider C, Dennehy C, Carter S. Exercise and cancer recovery. Champaign: Human Kinetics; 2003.

21. Thompson HJ, Zhu Z, Jiang W. Dietary energy restriction in breast cancer prevention. J Mammary Gland Biol Neoplasia 2003; 8: 133-142.

22. Winningham ML, MacVicar MG. The effect of aerobic exercise on patient reports of nausea. Oncol Nurs Forum 1988; 15: 447-450.

23. Rock CL, Demark-Wahnefried W. Nutrition and survival after the diagnosis of breast cancer: a review of the evidence. J Clin Oncol 2002; 20: 3302-3316.

24. Tretli S, Haldorsen T, Ottestad L. The effect of pre-morbid height and weight on the survival of breast cancer patients. Br J Cancer 1990; 62: 299-303.

25. Winningham ML, MacVicar MG, Bondoc M, Anderson JI, Minton JP. Effect of aerobic exercise on body weight and composition in patients with breast cancer on adjuvant chemotherapy. Oncol Nurs Forum 1989; 16: 683-689.

26. Schwartz AL. Fatigue mediates the effects of exercise on quality of life. Qual Life Res 1999; 8: 529-538.

27. Schwartz AL. Daily fatigue patterns and effect of exercise in women with breast cancer. Cancer Pract 2000; 8: 16-24.

28. Schwartz AL, Mori M, Gao R, Nail LM, King ME. Exercise reduces daily fatigue in women with breast cancer receiving chemotherapy. Med Sci Sports Exerc 2001; 33: 718-723.

29. Mock V, Pickett M, Ropka ME, Muscari LE, Stewart KJ, Rhodes VA, et al. Fatigue and quality of life outcomes of exercise during cancer treatment. Cancer Pract 2001; 9: 119-127.

30. Segal RJ, Reid RD, Courneya KS, Malone SC, Parliament $M B$, Scott CG, et al. Resistance exercise in men receiving androgen deprivation therapy for prostate cancer. J Clin Oncol 2003; 21: 1653-1659.

31. Anonymous. USDA dietary guidelines for Americans. Rockville: US Department of Health and Human Services. Home and Garden Bulletin No. 232:239; 2000.

32. Brown JK, Byers T, Doyle C, Coumeya KS, DemarkWahnefried W, Kushi LH, et al. Nutrition and physical activity during and after cancer treatment: an American Cancer Society guide for informed choices. CA Cancer J Clin 2003; 53: 268-291. 